\title{
MONITORAMENTO EM ÁREA DE CATASTROFE AMBIENTAL ATRAVÉS DE TÉCNICA DE DETECÇÃO DE MUDANÇAS
}

\author{
Jorgeane Schaefer dos Santos ${ }^{1}$, Christel Lingnau ${ }^{2}$
}

(recebido: 16 de março de 2010; aceito: 28 de outubro de 2012)

\begin{abstract}
RESUMO: O uso de imagens de satélites tem sido muito eficaz para o monitoramento da dinâmica do uso e ocupação do solo ao longo do tempo. Para isso, as técnicas de detecção de mudança têm sido fortes aliadas. Essas técnicas têm múltiplas complexidades dependendo do objetivo que se pretende alcançar. No presente estudo, buscou-se avaliar a aplicação da técnica de detecção de mudanças no monitoramento do uso e ocupação do solo nas áreas atingidas pela catástrofe ambiental de novembro de 2008 em SC, na região do Morro do Baú. Foram utilizadas 04 (quatro) imagens do sensor Landsat de datas distintas entre 1992 e 2009 (póscatástrofe). As imagens foram transformadas em índice de vegetação, com a utilização das bandas 7 e 4 para minimizar os efeitos atmosféricos e radiométricos. Máscara de sombreamento, utilizando o modelo digital do terreno, foi desenvolvida de modo a evitar falsas mudanças causadas por sombra. Concluiu-se que o georreferenciamento deve ser muito preciso na aplicação dessas técnicas de detecção de mudanças; que o índice de vegetação com bandas 7 e 4, bem como a máscara de sombreamento, foram eficazes na minimização de falsas mudanças e; que as técnicas aplicadas mostraram-se eficientes para detectar mudanças ocorridas nas áreas atingidas pela catástrofe.
\end{abstract}

Palavras-chave: Bacia do Itajaí, monitoramento uso do solo, deslizamentos de terra.

\section{MONITORING ENVIRONMENTAL CATASTROPHE AREA THROUGH CHANGE DETECTION TECHNIQUES}

\begin{abstract}
The use of satellite images has been very effective for monitoring the dynamics of the land use and occupation over time. For this purpose, the change detection techniques have been strong allies. These techniques have multiple complexities depending on the objective to be achieved. This study aims to evaluate the technique for land use and land cover changing detection in areas affected by the environmental disaster of November 2008 in the region of Morro do Baú, Santa Catarina, Brazil. A total of 04 (four) images from different dates between 1992 and 2009 (post-disaster) were used. The images were processed in vegetation index using bands 7 and 4 in order to minimize atmospheric and radiometric distortions. Shadow mask, construted from the digital terrain model, was developed to avoid false changes caused by shade. It was concluded that the georeferencing must be very accurate in applying these techniques. The vegetation index by using bands 7 and 4and the shadow mask, were effective in minimizing false changes. It showed that the techniques applied are effective to detect changes in areas affected by the disaster.
\end{abstract}

Key-words: Itajaí wattershed, land use monitoring, landslides.

\section{INTRODUÇÃO}

A bacia do Itajaí compreende municípios de grande importância para o Estado de Santa Catarina, sendo especialmente um pólo industrial de destaque do estado. Essa bacia tem sofrido com os problemas causados por enchentes, erosões, poluição, assoreamento, inadequado planejamento urbano (SCHAEFER-SANTOS, 2003) e, mais recentemente, em novembro de 2008 , a região do médio Itajaí foi afetada por uma catástrofe ambiental nunca antes ocorrida na região, com inúmeros deslizamentos de terra, soterramentos e mortes. Ao longo do tempo, com o êxodo rural crescente, a população das cidades pólo cresceu e a ocupação se deu nas encostas e fundos de vale desta região bastante montanhosa.

O monitoramento do uso e ocupação do solo por meio de imagens de satélite se dá pela aplicação de técnicas de detecção de mudanças as quais constituem na análise de um par de imagens adquiridas da mesma área geográfica, em datas diferentes, de modo a identificar possíveis alterações ocorridas (BOVOLO; BRUZZONE, 2005). A relativa disponibilidade de imagens bem como o aprimoramento das técnicas de interpretação, facilitam o mapeamento de mudanças temporais de pequena, média ou larga escala de tempo e/ou espaço.

${ }^{1}$ Engenheira Florestal, Doutoranda em Engenharia Florestal - Universidade Federal do Paraná/UFPR - Programa de Pós-graduação em Engenharia Florestal - Av. Pref. Lothário Meissner, 900, Jardim Botânico - Campus III - 80210-170 - Curitiba, PR, Brasil eng_jorgeane@yahoo.com.br

${ }^{2}$ Engenheira Florestal, Professora Doutora em Engenharia Florestal - Universidade Federal do Paraná/UFPR - Departamento de Ciências Florestais - Av. Pref. Lothário Meissner, 900, Jardim Botânico - Campus III - 80210-170 - Curitiba, PR, Brasil - lingnau@ufpr.br 
Os algoritmos para a detecção de mudanças em imagens de satélite são muitos e foram evoluindo com o passar do tempo. Eles vão dos mais simples aos mais complexos dependendo da necessidade e dos objetivos de cada estudo (CARVALHO JÚNIOR; SILVA, 2007). São vários os exemplos de aplicação de detecção de mudanças citados em Lu et al. (2004): Prakash e Gupta (1998) usou, diferença de imagens, razão de imagens e diferença de NDVI na detecção de mudanças de uso do solo em uma área de recife de corais na India e concluiu que não existem diferenças entre os métodos analisados; Haynes e Sader (2001) compararam diferenças de NDVI, Análise de Componentes Principais e composição colorida vermelho, verde e azul (RGB)-NDVI para a detecção de mudanças em clareiras florestais e regeneração florestal na Guatemala e perceberam que o método RGB-NDVI produziu os melhores resultados.

Muitas técnicas de detecção de mudança, tais como técnicas de álgebra e de categorias de transformação, requerem a seleção de algoritmos de limiaridade para a definição de "mudança" e "não-mudança" (FUNG; LEDREW, 1988 citados por LU et al., 2004). Lu et al. (2004) classificam as técnicas de detecção de mudanças em 7 (sete) categorias: (1) álgebra; (2) transformação; (3) classificação; (4) modelos avançados; (5) abordagens em Sistema de Informações Geográficas; (6) análises visuais; e (7) outras abordagens. Essas categorias são classificadas também quanto ao nível de complexidade.

Para avaliar a dinâmica de uso e ocupação do solo nas áreas atingidas pelos deslizamentos, foi realizada análise multitemporal de imagens do satélite Landsat 5 TM, objetivando testar o método de diferenca simples para detectar mudancas em paisagens com relevo acidentado. Como objetivos específicos, o estudo visou a identificar mudanças espaço-temporais na cobertura florestal, assim como identificar as áreas de deslizamentos de terra da catástrofe ambiental de novembro de 2008. O mapeamento de áreas de catástrofe ambiental, por meio da detecção de mudanças, pode se tornar uma ferramenta de indicação de cenários frágeis e propícios a novos eventos.

\section{MATERIAL E MÉTODOS}

\section{1 Área de Estudo}

A área de estudo situa-se entre as latitudes $26^{\circ} 20^{\prime} \mathrm{e}$ $27^{\circ} 50^{\prime}$ e longitudes $48^{\circ} 40^{\prime}$ e $50^{\circ} 20^{\prime}$ no curso médio do rio Itajaí, e compreende a sub-bacia do Médio Itajaí e parte da sub-bacia do Rio Luis Alves, englobando as áreas da catástrofe de novembro de 2008. O relevo da região é caracterizado por intensa dissecação do Rio Itajaí com vales profundos com encostas separadas por cristas bem marcadas na paisagem. Os processos erosivos, principalmente nas encostas desmatadas, ocasionam movimentos de massa, uma vez que o material resultante da alteração da rocha pode atingir até $20 \mathrm{~m}$ de profundidade (Gabinete de Planejamento do Estado de Santa Catarina GAPLAN, 1986).

\subsection{Base de Dados}

$\mathrm{O}$ estudo foi desenvolvido com base em imagens Landsat 5 TM, entre o periodo de 1992 e 2008 (Tabela 1). A base cartográfica consiste em cartas planimétricas na escala 1:50.000 e do modelo digital de elevação (MDE) produzido por meio de projeto conjunto entre agência espacial americana (NASA) e a agência de inteligência geoespacial (NGA) e disponibilizado pela Epagri em sua Mapoteca Digital (http://ciram.epagri.rct-sc.br).

Para que não sejam detectadas mudanças inexistentes, é necessário sanar problemas de ruídos de georreferenciamento, por meio de corregistro das imagens com o mínimo de erro admissível; ruídos de diferença de reflectância, pela minimização dos efeitos fenológicos de datas diferentes e das diferenças radiométricas; e diferenças de sombreamento entre imagens. Dessa forma, a seguir são descritos os procedimentos metodológicos sobre os aspectos citados acima.

Tabela 1 - Especificações técnicas das imagens de satélite utilizadas no estudo.

Table 1 - Technical specification of the satellite images used in the study.

\begin{tabular}{lcccc}
\hline \multirow{2}{*}{ Especificações técnicas } & \multicolumn{3}{c}{ Imagens } \\
\cline { 2 - 5 } & $\mathrm{t}_{1}$ & $\mathrm{t}_{2}$ & $\mathrm{t}_{3}$ & $\mathrm{t}_{4}$ \\
\hline Data de Passagem & $10 / 6 / 1992$ & $30 / 6 / 1999$ & $4 / 6 / 2007$ & $1 / 2 / 2009$ \\
Tempo Central (GMT) & $12: 35: 10$ & $12: 49: 29$ & $13: 05: 58$ & $12: 57: 14$ \\
Azimuth Sol $\left({ }^{\circ}\right)$ & 41,14 & 38,8352 & 34,7336 & 81,5054 \\
Elevação do Sol $\left({ }^{\circ}\right)$ & 26,6324 & 28,0045 & 31,5528 & 53,8078 \\
\hline
\end{tabular}

Cerne, Lavras, v. 19, n. 1, p. 133-140, jan./mar. 2013 


\subsection{Modelo de Sombreamento do Relevo}

A geração da máscara de sombra faz-se necessário em estudos multitemporais, sobretudo em áreas de relevo acidentado. Áreas podem ser detectadas como alteradas, em razão do fator de sombreamento diferente que ocorre entre as imagens. Para a geração da máscara de sombra, foram inseridas as informações relativas à imagem com a menor inclinação do sol, representada em sua data de aquisição. Essa máscara foi sobreposta às imagens classificadas sendo eliminadas as falsas mudanças.

\subsection{Processamento das Imagens}

\subsubsection{Georreferenciamento das imagens}

$\mathrm{Na}$ aplicação da detecção de mudanças usando várias imagens de datas diferentes de uma mesma área, a correpondência geográfica pixel a pixel deverá ser muito criteriosa. Segundo Bruzzone e Cossu (2002, 2003) e Bruzzone et al. (2007), ao se comparar duas imagens para a detecção de mudanças, faz-se primordial que seja feito corregistro das imagens. O método utilizado para a correção geométrica foi o do Vizinho Mais Próximo, aplicando um polinômio de segunda ordem. No georreferenciamento em questão foi encontrado um erro de 0,25 a 0,37 pixel.

\subsubsection{Correção radiométrica e atmosférica}

Segundo Ponzoni e Santos (2008), quando os dados a serem analisados provêm de sensores diferentes, torna-se obrigatória a normalização radiométrica. Hall et al. (1991) afirmam que a correção radiométrica se justifica dentro das bandas do visível, pois os algoritmos da correção radiométrica ajustam a reflectância da superfície para os efeitos das diferenças relativas da atmosfera dentro de $1 \%$, mas, as bandas do infra-vermelho próximo. médio sofrem menor influência da atmosfera. Assim sendo, no presente estudo, sendo que todas as imagens analisadas pertencem ao mesmo sensor e optou-se por utilizar as bandas 4 e 7 no índice de vegetação, a aplicação de algoritmos de correção radiométrica não se faz necessária.

\subsection{3 Índice de Vegetação (IV)}

O comportamento espectral da vegetação nos produtos de sensoriamento remoto responde a fatores inerentes à planta e outros ao meio, tais como clorofila, estrutura celular, quantidade de água nas folhas, tipologia florestal, densidade do dossel, entre outros (HILDEBRANDT, 1985; PONZONI, 2002). O índice de vegetação normalizado (NDVI - Normalized Difference Vegetation Index) traduz o "verdor" de uma imagem de satélite (BACA, 2005), pois, ao fazer a diferença entre as bandas do infravermelho próximo e do vermelho, evidenciase a absorção de luz solar pela clorofila e também ao espalhamento que a radiação eletromagnética sofre no interior da folha. À medida que uma floresta se adensa, na região do visível a reflectância cai, por outro lado, na região do infravermelho próximo, a reflectância aumenta. Ambos quase em relação exponencial, segundo Ponzoni (2002). Todavia, foi utilizada, neste estudo, nova proposta de índice de vegetação (IV) com as bandas 4 e 7, a qual evidenciou as diferenças existentes na área bem e efeitos atmosféricos não são observados nas bandas 4 e 7 .

Foram utilizados no IV (Equação 1) a banda do infravermelho próximo(B4, $0.76-0.90 \mu \mathrm{m})$ e do infravermelho médio(B7, $2.08-2.35 \mu \mathrm{m})$.

$$
\begin{aligned}
& \mathrm{IV}=(\mathrm{B} 4-\mathrm{B} 7) /(\mathrm{B} 4+\mathrm{B} 7) \\
& \text { Onde: } \\
& \mathrm{B} 4=0.76-0.90 \mu \mathrm{m} \\
& \mathrm{B} 7=2.08-2.35 \mu \mathrm{m}
\end{aligned}
$$

\subsection{Análise de Detecção das Mudanças}

O objetivo da aplicação das técnicas de detecção de mudanças é, dentro de um conjunto de pixels, identificar aqueles que são significantemente diferentes entre a primeira e a última imagem da sequência (RADKE et al., 2005). Seleciona-se a imagem inicial $\left(\mathrm{t}_{1}\right)$ e a imagem final $\left(\mathrm{t}_{2}\right)$, sendo que o procedimento é repetido entre os pares " $\mathrm{t}_{2}-\mathrm{t}_{1}$ ", " $\mathrm{t}_{3}-\mathrm{t}_{2}$ " e " $\mathrm{t}_{4}-\mathrm{t}_{3}$ ", gerando três imagens classificadas (Detecção de Mudanças 1 - DM 1 , Detecção de Mudanças $2-\mathrm{DM}_{2}$ e Detecção de Mudanças $3-\mathrm{DM}_{3}$ ). A classificação do presente estudo seguiu valores de mudança entre -5 e +5 . Mudanças próximas a zero (entre $2 \mathrm{a}+2$ ) foram ignoradas, representando "não-mudança", e "mudança abrupta" foram identificadas como acréscimo ou redução da biomassa. O método de diferenças simples e o percentual de diferença entre os IVs em quatro momentos $\left(\mathrm{t}_{1}, \mathrm{t}_{2}, \mathrm{t}_{3}\right.$ e $\left.\mathrm{t}_{4}\right)$ foi utilizado, como segue:

$D M(x)=t_{2}(x)-t_{1}(x)$

Onde:

$D M(\mathrm{x})=$ imagem classificada por diferença simples;

$t_{2}(\mathrm{x})=$ índice de vegetação no tempo final da análise;

$t_{1}(\mathrm{x})=$ índice de vegetação no tempo inicial da análise.

A imagem obtida, pós-classificação, chamada de máscara de mudança (RADKE et al. 2005), é gerada de acordo com as classes de limiaridade, identificadas pelo analista:

Cerne, Lavras, v. 19, n. 1, p. 133-140, jan./mar. 2013 
$B(x)=\left\{1 \stackrel{\text { se }}{\longrightarrow} D(x) \geq \tau_{n} ;\right.$

$B(x)=\{0 \stackrel{\text { se }}{\longrightarrow}<\tau$

Onde:

$B(x)=$ máscara de mudança

$\tau_{n}=$ limiaridade em $\mathrm{n}$ classes.

O valor para as classes de limiaridade foi definido a partir dos valores de desvio padrão das imagens do índice de vegetação(LUet al., 2004; RICHARDS; JA, 2006), sendo que, para o presente estudo, foi adotado o desvio padrão médio dos IV's de 0,20 para um total de 11 classes de limiaridade.

\section{RESULTADOS E DISCUSSÃO}

\subsection{Processamento das imagens}

\subsection{1 Índice de Vegetação}

No detalhamento de cada área afetada pelos deslizamentos do evento de novembro de 2008, o valor do índice de vegetação entre $t_{1}, t_{2}$ e $t_{3}$, é semelhante. No entanto, nos locais em que ocorreram os deslizamentos e a deposição dos sedimentos carreados, o valor do IV diminui até muito próximo a 0,0 em t . As áreas afetadas pelos deslizamentos são facilmente identificadas (cinza escuro) e são reconhecidas nas imagens analisadas (Figura 1).

\subsubsection{Detecção de Mudanças}

O fator de limiaridade das classes de detecção de mudanças adotado foi o desvio padrão médio 0,20 dos índices de vegetação obtidos das imagens da área de estudo como um todo. Para fins dessa análise, restringese, no entanto, apenas a uma pequena área da bacia, na qual ocorreram muitos deslizamentos, totalizando 11.215 ha. As 11 classes encontradas foram reagrupadas, conforme Tabela 2. As mudanças próximas a zero $(-2 \mathrm{a}+2)$ foram eliminadas da análise, sendo, no entanto incluídas no histograma apenas para a compreensão da classificação global de mudanças.

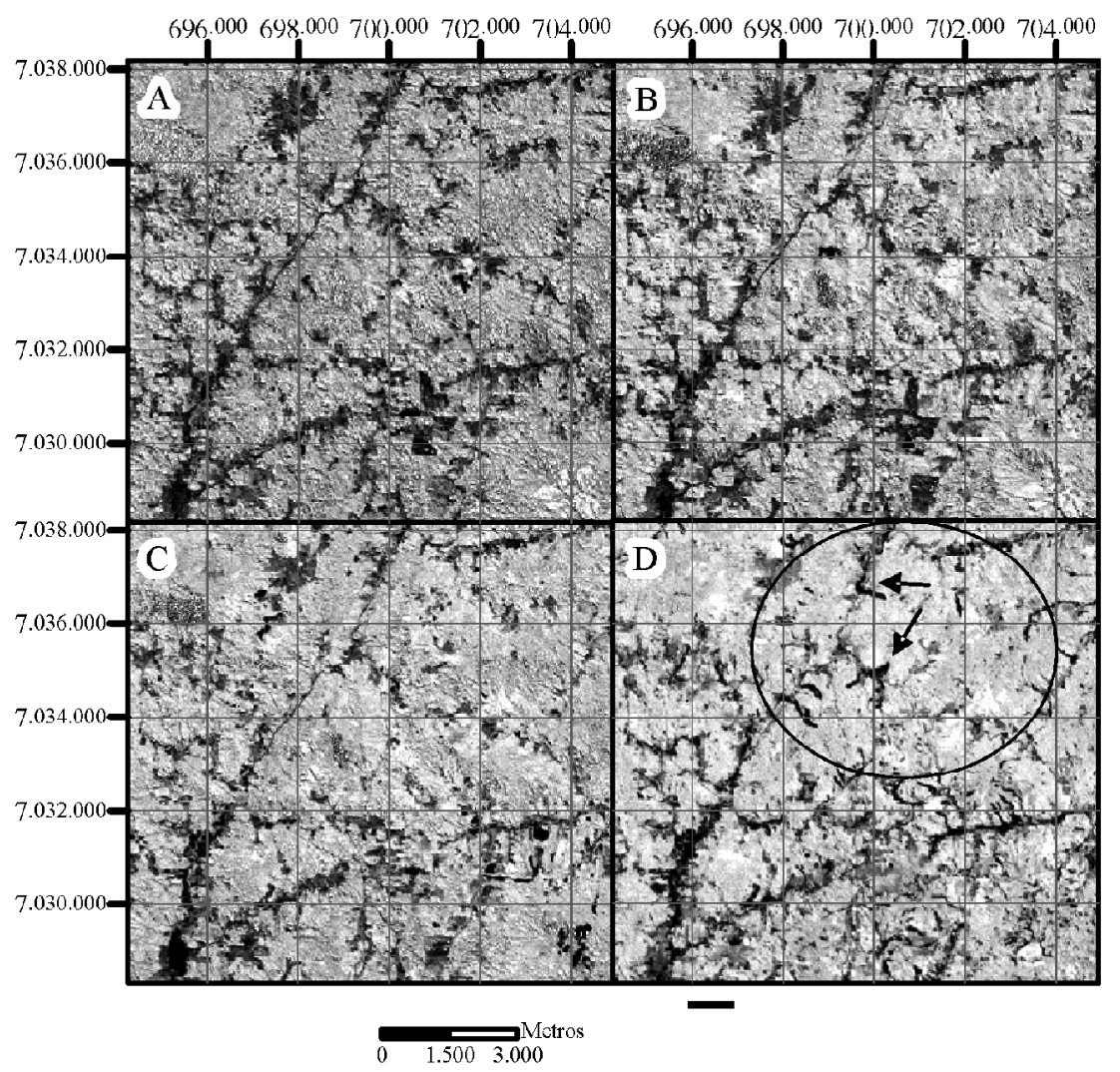

Figura 1 - Índice de vegetação para as 4 datas. A - 1992; B - 1999; C - 2007 e D 2009.

Figure 1-Vegetation index for the 4 dates. A-1992; B - 1999; C- 2007 and D - 2009.

Cerne, Lavras, v. 19, n. 1, p. 133-140, jan./mar. 2013 
Os histogramas de $\mathrm{DM}_{1}, \mathrm{DM}_{2}$ e $\mathrm{DM}_{3}$ (Figura 2) revelam que a maior parte das mudanças, ocorreu ao redor do eixo zero, inidicando a ocorrência de mudanças pouco expressivas. $\mathrm{O}$ acréscimo de biomassa pode ser observado na sequência dos histogramas (Figura 2), sendo que entre A (1992) e D (2009) ocorreu aumento da biomassa florestal.

As áreas de deslizamentos de terra e com deposição de sedimentos locais de forte movimentação de terra, foram registrados como redução moderada a severa (classe de mudança -4 e -5$)$.

\subsection{Análise de Detecção das Mudanças}

A aplicação da máscara de sombreamento promoveu a eliminação de falsas mudanças na área de estudo, especialmente quando referentes ao acréscimo da biomassa. Na bacia do Itajaí, como um todo, tem ocorrido aumento da ocupação das áreas por florestas (VIBRANS, 2003), o mesmo processo foi identificado na área de estudo (SCHAEFER-SANTOS, 2003), especialmente em razão do abandono de áreas agrícolas e pastagens.

Tabela 2 - Reclassificação das imagens $\mathrm{DM}_{1}, \mathrm{DM}_{2}$ e $\mathrm{DM}_{3}$.

Table 2 - Re-classification of the images $C D_{1}, C D_{2}$ e $C D_{3}$.

\begin{tabular}{ccc}
\hline Classes Change Detection & Valores & Classes de mudança de biomassa \\
\hline Change (+5): & $>0,80$ & Acréscimo severo \\
Change (+4): & 0,60 a 0,80 & Acréscimo moderado \\
Change (+3): & 0,40 a 0,60 & Acréscimo leve \\
Change (+2): & 0,20 a 0,40 & \\
Change (+1): & 0 a 0,20 & \\
No Change: & 0,0 a 0,0 & Sem mudanças \\
Change (-1): & 0,0 a $-0,20$ & \\
Change (-2): & $-0,20$ a $-0,40$ & Redução leve \\
Change (-3): & $-0,40$ a $-0,60$ & Redução moderada \\
Change (-4): & $-0,60$ a $-0,80$ & Redução severa \\
Change (-5): & $<-0,80$ & \\
\hline
\end{tabular}

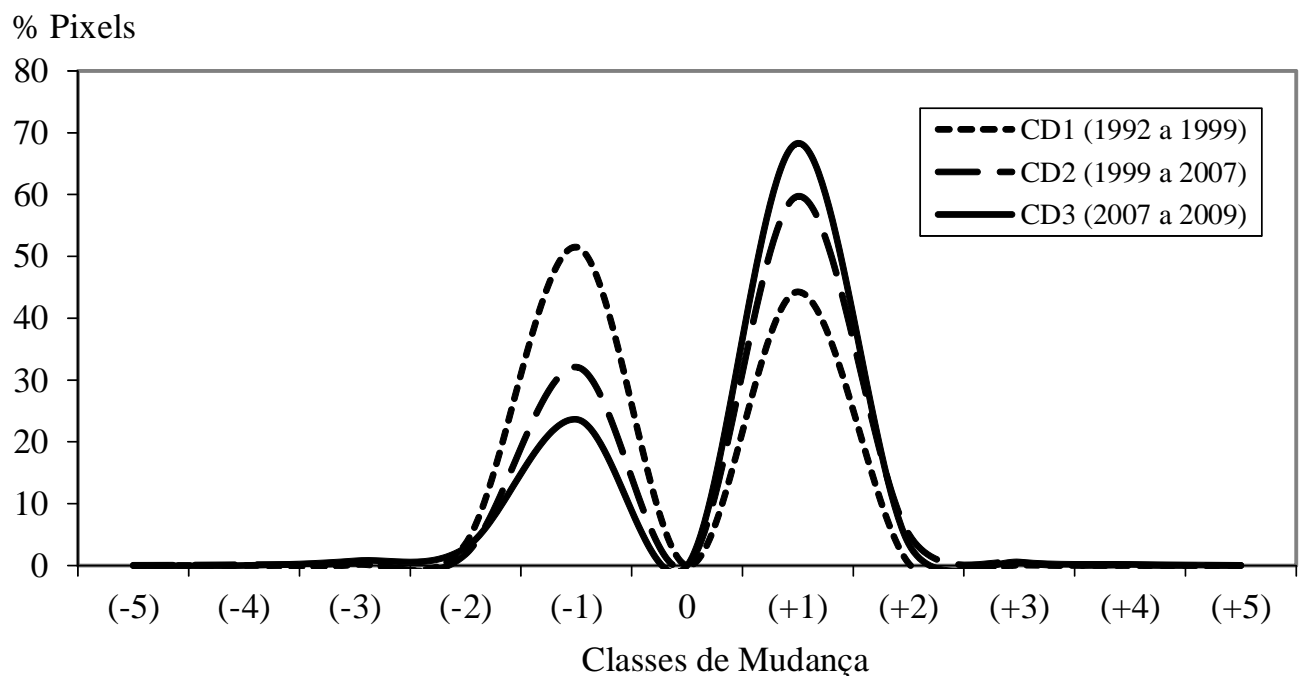

Figura 2 - Histograma das imagens da Detecção de Mudanças.

Figure 2 - Histogram of Change Detection images. 
Confrontando a interpretação dos IV's (Figura 3), nota-se que ocorreu aumento da biomassa florestal ao longo do tempo. Em $\mathrm{DM}_{1}$, conforme também notado pelo histograma, perdas e ganhos de biomassa se equivalem. Porém, especialmente observado em $\mathrm{DM}_{1}$ e $\mathrm{DM}_{3}$ há ganho de biomassa, exceto, nesta última, nas áreas de deslizamento ocorridas em novembro de 2008 (Figura 3). Os deslizamentos foram registrados pela classe 'redução leve da biomassa' justificada pelos deslizamentos de terra nas áreas que eram ocupadas por florestas nas encostas, expondo o solo, essas mesmas áreas podem ser identificadas em toda a imagem $\mathrm{IV}_{4}$, detectadas na imagem $\mathrm{DM}_{3}$ (Figura 3).

No período analisado em toda a área de estudo houve acréscimo de biomassa. Em se tratando das causas dos deslizamentos naquela área, não se afirma que os mesmos ocorreram em decorrência da exposição do solo ou da redução da biomassa nos anos estudados.

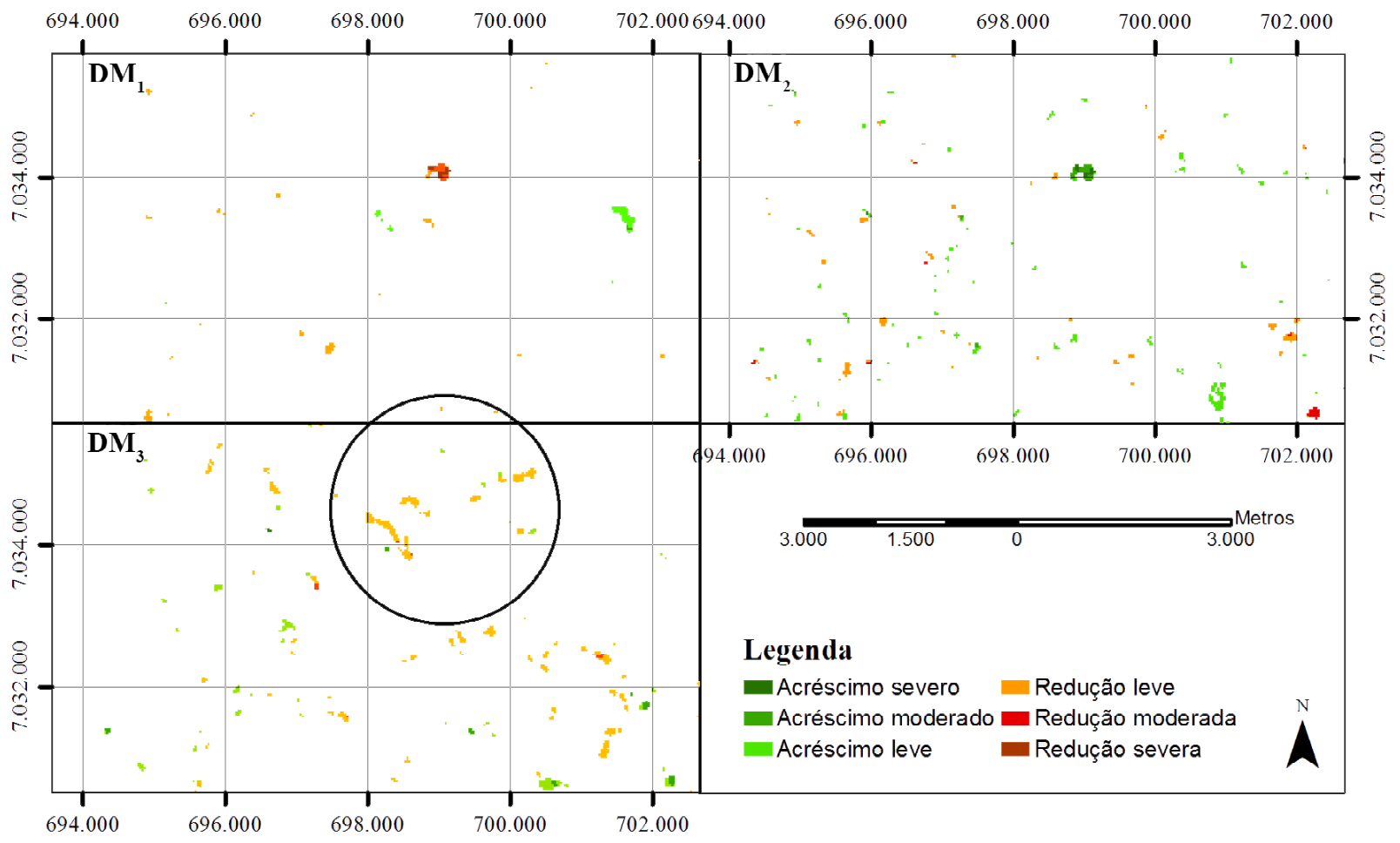

Figura 3 - Imagens da Detecção de Mudanças, com aplicação do modelo de sombreamento e no detalhe áreas em que ocorreram deslizamentos.

Figure 3 - Change Detection Images with application of the shadow model and landslides areas in detail.

\section{CONCLUSÕES}

As conclusões gerais deste estudo foram:

- O índice de vegetação gerado a partir das bandas 7 e 4 mostrou-se eficiente por não apresentar efeitos atmosféricos que possam representar falsas mudanças.

- Faz-se necessário aplicar máscara de sombreamento quando da aplicação da análise de detecção de mudanças em áreas com relevo acidentado. A máscara aplicada neste artigo mostrou-se eficiente para eliminar falsas mudanças relacionadas ao sombreamento do terreno.

- O georreferenciamento das imagens com precisão é fundamental para evitar a detecção de falsas mudanças.

- O desmatamento não é justificativa isolada para os deslizamentos ocorridos naquele local, uma vez que as áreas de deslizamento apresentavam cobertura florestal na série observada. A intensidade do evento pluviométrico

Cerne, Lavras, v. 19, n. 1, p. 133-140, jan./mar. 2013 
pode ter sido o fator mais determinante associado aos deslizamentos de terra na área estudada.

- A análise de detecção de mudanças por diferença simples aplicadas aos índices de vegetação de imagens Landsat 5 TM, tornou-se eficiente para identificar as áreas de deslizamentos de terra e de deposição de solo carreado na catástrofe de novembro de 2008.

- Aliada aos sistemas de informações geográficas, a detecção de mudanças aplicada a imagens em séries temporais é ferramenta eficaz no controle de processos de dinâmicos de uso e ocupação do solo.

\section{AGRADECIMENTOS}

Agradecemos ao CNPq pela bolsa de estudos.

\section{REFERÊNCIAS}

BACA, J. F. M. Dinâmica do NDVI para América do Sul: 19812001. In: SIMPÓSIO BRASILEIRO DE SENSORIAMENTO REMOTO, 12., 2005, Goiânia. Anais... São José dos Campos: INPE, 2005. p. 3487-3494. 1 CD-ROM.

BOVOLO, F.; BRUZZONE, L. A detail-preserving scaledriven approach to change detection in multitemporal SAR images. IEEE Transaction on Geoscience and Remote Sensing, New York, v. 43, n. 12, p. 2963-2972, Dec. 2005.

BRUZZONE, L.; BOVOLO, F.; MARCHESI, S. A multiscale change detection technique robust to registration noise. In: GHOSH, R. K.; PAL, S. K. (Ed.). PReMI 2007, LNCS 4815. Berlin: Springer-Verlag, 2007. p. 77-86.

BRUZZONE, L.; COSSU, R. An adaptive approach to reducing registration noise effects in unsupervised change detection. IEEE Xplore Transaction on Geosciences and Remote Sensing, Ann Arbor, v. 41, n. 11, p. 2455-2465, Nov. 2003.

BRUZZONE, L.; COSSU, R. An unsupervised change detection technique robust to registration noise. IEEE Xplore Transaction on Geoscience and Remote Sensing, Ann Arbor, v. 1, p. 306-308, 2002.

CARVALHO JÚNIOR, O. A.; SILVA, N. C. da. Detecção de mudança espectral uma nova metodologia para análise de séries temporais. In: SIMPÓSIO BRASILEIRO DE SENSORIAMENTO REMOTO, 13., 2007, Florianópolis. Anais... Florianópolis: INPE, 2007. p. 5635-5641.
GABINETE DE PLANEJAMENTO DO ESTADO DE SANTA CATARINA. Atlas de Santa Catarina. Rio de Janeiro: Aerofoto Cruzeiro, 1986. 173 p.

HALL, F. G.; STREBEL, D. E.; NICKESON, J. E.; GOETZ, S. J. Radiometric rectification: toward a common radiometric response among multidate, multisensor imagens. Remote Sensing of Environment, New York, v. 91, p. 90062-B, 1991.

HAYES, D. J.; SADER, S. A. Change detection techniques for monitoring forest clearing and regrowth in a tropical moist forest. Photogrammetric Engineering and Remote Sensing, Falls Church, v. 67, n. 9, p. 1067-1075, 2001.

HILDEBRANDT, G. Waldschaden und ihre Erfassung durch Fernerkundung. In: NIESSLEIN, E.; VOSS, G. (Ed.). Was wir über das Waldsterben wissen. Cologne: Deutscher Institutes Verlag, 1985. p. 97-111.

LU, D.; MAUSEL, P.; BRONDÍZIOS, E.; MORAN, E. Change detection techniques. International Journal of Remote Sensing, Basingstoke, v. 25, n. 12, p. 2365-2407, June 2004.

PONZONI, F. J. Sensoriamento remoto no estudo da vegetação: diagnosticando a mata atlântica. In: RUDORFF, B. F. T.; MORAES, E. C.; PONZONI, F. J.; CAMARGO JÚNIOR, H.; CONFORTE, J. C.; MOREIRA, J. C.; EPIPHANIO, J. C. N.; MOREIRA, M. A.; KAMPEL, M.; ALBUQUERQUE, P. C. G. de; MARTINI, P. R.; FERREIRA, S. H.; TAVARES JÚNIOR, S. S.; SANTOS, V. M. N. dos (Ed.). Curso de uso de sensoriamento remoto no estudo do meio ambiente. São José dos Campos: INPE, 2002. cap. 8, p. 27.

PONZONI, F. J.; SANTOS, S. B. Conversão de números digitais de imagens orbitais em valores de FRB de superfície. Boletim de Ciências Geodésicas, Curitiba, v. 14, n. 4, p. 541-556, out./dez. 2008.

PRAKASH, A.; GUPTA, R. P. Land-use mapping and change detection in a coal mining area: a case study of the Jharia coalfield, India. International Journal of Remote Sensing, Basingstoke, v. 19, p. 391-410, 1998.

RADKE, R. J.; AL-KOFAHI, O.; ROYSAM, B. Image change detection algorithms: a systematic survey. IEEE Transaction on Geoscience and Remote Sensing, New York, v. 14, n. 3, p. 294-307, Mar. 2005.

Cerne, Lavras, v. 19, n. 1, p. 133-140, jan./mar. 2013 
RICHARDS, J. A.; JIA, X. Remote sensing digital image analysis: an introduction. $4^{\text {th }}$ ed. Berlin: Springer-Verlag, 2006.

SCHAEFER-SANTOS, J. Ocupação do solo e comportamento hidrológico da sub-bacia do Rio Luis Alves, bacia do Rio Itajaí, Santa Catarina. 2003. 199 p. Dissertação (Mestrado em Engenharia Florestal) Universidade Federal do Paraná, Curitiba, 2003.

VIBRANS, A. C. A cobertura florestal da bacia do Rio Itajaí: elementos para uma série histórica. 2003. 239 p. Tese (Doutorado em Geografia) - Universidade Federal de Santa Catarina, Florianópolis, 2003. 